\title{
NOTES TOWARDS THE CONSTRUCTION OF NONLINEAR RELATIVISTIC QUANTUM FIELDS. II: THE BASIC NON- LINEAR FUNCTIONS IN GENERAL SPACE-TIMES
}

\author{
BY IRVING SEGAL ${ }^{1}$
}

Communicated by Murray Protter, September 17, 1969

1. In earlier work [1], [2], the generalized nonlinear functions which enter formally into certain nonlinear relativistic quantized partial differential equations in a two-dimensional space-time were given mathematical formulation and treatment. The theory of these highly singular functions, and in particular their locality, when combined with hyperbolicity ideas implemented by the use of the Lie-Trotter formula, gave a means of adapting the treatment of quantum field dynamics in terms of a group of automorphisms of a $C^{*}$-algebra, developed initially in [3], to the equations in question. The present work describes an extension of the theory of these basic functions (or "renormalized products of quantum fields"), to general space-times and laws of dependence of the energy on the momentum, relativistic theory in a Minkowski space of any dimension being a rather special case. The general dynamical implications of these results will be treated later.

2. Since apart from the recent work [1], [2], [4], [5], [6] there is little mathematical literature on renormalized powers of quantum fields, it may be helpful to describe briefly the background of the subject. Renormalized products of quantum variables in general, and of quantum fields in particular, arose and were studied soon after the introduction of quantum mechanics. The ambiguities in the calculus of operators satisfying "canonical commutation relations" became more serious in the case of quantum fields, where they appeared in part as "infinities" or so-called "divergences." The usage in the physical literature was standardized as a result of work of Wick [6], whose mathematical core as now perceived is a theorem in finite-dimensional algebra (cf. Theorem 1.3 of [2]). Its extensive application in the physical literature has been to situations involving a continuum of variables (typically, the "values" of a generalized function on $R^{n}$ ); these are treated by formal analogy with the finitedimensional, purely algebraic, situation.

AMS Subject Classification. Primary 2846, 8147; Secondary 3495, 4665.

Key Words and Phrases. Renormalized product, regularity domain, temporal smoothing, quantum field.

${ }^{2}$ Research sponsored in part by the Office of Scientific Research. 
The germ of a different approach to the definition and treatment of renormalized products of quantum fields was noted in [7]. This approach, unlike that of Wick which was applicable only to "free fields," could be applied in principle to arbitrary fields such as the putative "interacting" field. It was in the direction of an intrinsic characterization based on the elementary notions;

(a) the formation of a renormalized product of generalized function should be a local operation on the functions;

(b) the usual laws of algebra, such as the conclusion of the binominal theorem, should remain formally valid for the renormalized operations;

(c) the only formal difference from the conventional product (in general, nonexistent in a mathematical sense) should be the vanishing of the expectation values of the renormalized products.

The analytical viability of this approach was first established in [1], [2] by connecting it with another approach to renormalization based on functional integration ideas and their application to the representation of quantum fields. In order for an evolutionary nonlinear partial differential equation to be well-defined, it has appeared essential that the nonlinear terms be defined for each fixed time; as a result, smoothing with respect to space is admissible in the nonlinear terms, but not smoothing with respect to time (or space-time); since, in quantum field theory, smoothing with respect to time is much more powerful than smoothing with respect to space, this is a serious limitation. In two space-time dimensions, the fixed-time renormalized powers of a scalar field are representable by random variables on a probability space, and the simultaneous diagonalization of the renormalized powers of the field thereby obtained. In higher-dimensional space-times, the fixed-time renormalized powers are now perceived to be generalized operators which typically map every vector in their domains outside of the Hilbert space in question. The functional integration approach is consequently not directly applicable, and a different one is used in [3] to formulate and establish for rather general cases results which are formally identical to those for the two-dimensional case. On the other hand, these results, once established, have implications for the functional integration interpretation, and lead for example to a natural and invariant definition for the (renormalized) powers of a "white noise" on an arbitrary abelian group.

Mention may also be made of still another mathematical approach to renormalized products of free fields in [8]. This work, utilizing on an ad hoc basis a definition of renormalization due to Caienello, 
established corresponding renormalized products in a scalar quantum field as operator-valued distributions on space-time. The operators in question were defined on a domain which is dense in the basic Hilbert space, but otherwise sufficiently small as to avoid nontrivial involvement with infinite-degree-of-freedom considerations. In particular, it is not invariant under the complex exponentials of the fields which are relevant to a comprehensive analytical treatment, for reasons similar to those involved in the transition from the Heisenberg to the Weyl relations; indeed, a nonlinear variant of the Weyl relations applicable to the renormalized powers is used in the present work.

3. Apart from the foregoing introduction and an appended section briefly correlating the present terminology and results with colloquial usage and heuristic theory, the presentation here will be strictly mathematical. This section treats notation and preliminary technicalities.

If $L$ is a given complex Hilbert space, the corresponding normal symmetric quantum process $\Omega(L)=(K, W, \Gamma, v)$ consists of:

(1) a complex Hilbert space $K$;

(2) a continuous mapping $W$ from $L$ to the unitary operators on $K$ satisfying the Weyl relations:

$$
W(z) W\left(z^{\prime}\right)=\exp \left[(i / 2) \operatorname{Im}\left(\left\langle z, z^{\prime}\right\rangle\right)\right] W\left(z+z^{\prime}\right) ;
$$

(3) a continuous representation $U \rightarrow \Gamma(U)$ of the group of all unitary operators on $H$ by similar operators on $K$, having the properties that

(a) $\Gamma(U) W(z) \Gamma(U)^{-1}=W(U z)$ for all $U$ and $z$;

(b) $d \Gamma(X) \geqq$ for any selfadjoint operator $X$ in $L$ such that $X \geqq 0$, where $d \Gamma(X)$ denotes the selfadjoint generator of the oneparameter group $\Gamma\left(e^{i t X}\right), t \in R^{1}$;

(4) a unit vector $v \in K$ which is cyclic for the $W(z)$ and such that $\Gamma(U) v=v$ for all $U$.

The foregoing properties determine $\Omega(L)$ uniquely, within unitary equivalence. The selfadjoint generator of the one-parameter unitary group [ $\left.W(t z): t \in R^{1}\right]$ will be denoted as $\Psi(z)$.

For any operator $T$ in a real or complex Hilbert space $H^{\prime}, D(T)$ will denote the domain; $D_{\infty}(T)$ will denote the common part of the domains of the $T^{n}$ when $n$ ranges over the positive integers; $[D(T)]$ will denote the completion of $D(T)$ as a Hilbert space relative to the inner product $\langle x, y\rangle_{T}=\langle T x, T y\rangle_{;}$and $\left[D_{\infty}(T)\right]$ will denote $D_{\infty}(T)$ as 
a topological linear space, a generic neighborhood of 0 consisting of all $x$ such that $\left\|T^{k} x\right\|<\epsilon$ for $k<k_{0}$, for some $\epsilon$ and $k_{0}$.

$G$ will denote a given locally compact abelian group; $B$ a given positive selfadjoint translation invariant operator in $L_{2}(G) ; C$ the restriction of $B^{1 / 2}$ to the real Hilbert space $H^{\prime}$ consisting of the real elements of $L_{2}(G)$; and $H$ the complex Hilbert space which as a real linear space is $[D(C)] \oplus\left[D\left(C^{-1}\right)\right]$; whose complex structure and fundamental sesquilinear form are given by the following equations, where $z=x \oplus y$ and $z^{\prime}=x^{\prime} \oplus y^{\prime}$ are arbitrary in $H: i: x \oplus y \rightarrow-B^{-1} y \oplus B x ;\left\langle z, z^{\prime}\right\rangle$ $=\left\langle C x, C x^{\prime}\right\rangle+\left\langle C^{-1} y, \quad C^{-1} y^{\prime}\right\rangle+i\left(\left\langle x, y^{\prime}\right\rangle-\left\langle x^{\prime}, y\right\rangle\right)$. The selfadjoint generator of the one-parameter unitary group $U(t)$ in $H$ defined by the equation: $U(t) z=z^{\prime}$, with $x^{\prime}=\cos (t B) x+(\sin (t B) / B) y, \quad y^{\prime}$ $=-B \sin (t B) x+\cos (t B) y$, will be denoted as $A$; and $d \Gamma(A)$ will be denoted as $H . \Psi(C x)$ will be denoted as $\Phi(x)$, and $\Psi\left(i C^{-1} x\right)$ as $\dot{\Phi}(x)$. The spectral function for $B$ on $G^{*}$ will be denoted as $B(\cdot)$.

4. The unicity of the renormalized products as characterized in Theorem 2 follows from a result applicable to an abstract space $H^{\prime}$.

TheOREM 1 [5]. Let $A^{\prime}$ be a given selfadjoint operator in a complex Hilbert space $H^{\prime}$ such that $A^{\prime} \geqq \epsilon I$ for some $\epsilon>0$. and let $H^{\prime}=d \Gamma\left(A^{\prime}\right)$. Then the mapping $(z, u) \rightarrow W(z) u$ is infinitely differentiable from $\left[D_{\infty}\left(A^{\prime}\right)\right] \times\left[D_{\infty}\left(H^{\prime}\right)\right]$ into $\left[D_{\infty}\left(H^{\prime}\right)\right]$; and any continuous sesquilinear form on $\left[D_{\infty}\left(H^{\prime}\right)\right]$ which is invariant under the $W(z)$ for all $z \in D\left(A^{\prime}\right)$ is proportional to the fundamental form on $K$.

An analogous result for unitary representations of Lie groups has been established by N. S. Poulsen (Massachusetts Institute of Technology, Mathematics Department).

For simplicity, only renormalized powers, rather than the similarly treated renormalized products involving suitably defined derivatives, are considered in

TheOREM 2 [5]. If $B(\cdot)^{-1} \in L_{p}\left(G^{*}\right)$ for all sufficiently large $p$, then there exist for $n=0,1, \cdots$, unique functions $\phi_{n}(\cdot)$ from $G$ to the continuous sesquilinear forms on $\left[D_{\infty}(H)\right]$ such that

(i) themapping $\left(a, u, u^{\prime}\right) \rightarrow \phi_{n}(a)\left(u, u^{\prime}\right)$ iscontinuousfrom $G \times\left[D_{\infty}(H)\right]$ $\times\left[D_{\infty}(H)\right]$ into $C$, for every $n$;

(ii) $\phi_{0}(a)\left(u, u^{\prime}\right)=\left\langle u, u^{\prime}\right\rangle$ for arbitrary $u, u^{\prime} \in D_{\infty}(H), \phi_{n}(a)(v, v)=0$ for arbitrary $a \in G$ and $n>0$;

(iii) For all $a \in G$, real $f \in D_{\infty}(B)$, and $u$ and $u^{\prime}$ in $D_{\infty}(H)$; and with $x=f \oplus 0 ; \phi_{n}(a)\left(e^{i \Phi(x)} u, e^{i \Phi(x)} u^{\prime}\right)=\phi_{n}(a)\left(u, u^{\prime}\right) ; \phi_{n}(a)\left(e^{i \dot{\Phi}(x)} u, e^{i \dot{\Phi}(x)} u^{\prime}\right)$ $=\sum_{r=0}^{n}\left(\begin{array}{l}n \\ r\end{array}\right) x(a)^{r} \phi_{n-r}(a)\left(u, u^{\prime}\right)$. 
In this result the operator $B$ plays two analytically distinct roles: one in connection with the definition of the regularity domains $\left[D_{\infty}(A)\right]$ and $\left[D_{\infty}(H)\right]$; the other in connection with the definition of the processes $\Phi$ and $\dot{\Phi}$. A more general result involving two operators may here be represented by essentially the case in which $B$ is replaced by $I$ in the second connection; this provides an intrinsic characterization of renormalized powers of the so-called "white noise," which is more singular than any relativistic quantum process. The relation between Theorems 2 and 3 may be clarified by the observation that the mapping $x \oplus y \rightarrow C x \ominus i C^{-1} y$ is unitary from $H$ onto $L_{2}(G)$, and transforms $A$ into $B$.

TheOREM 3 [6]. If $L^{\prime}=L_{2}(G)$, if $B$ is as in Theorem 2, and if $\boldsymbol{K}$ $=d \Gamma(B)$, then there exist for $n=0,1, \cdots$ unique functions $\phi_{n}(\cdot)$ from $G$ to the continuous sesquilinear forms on $\left[D_{\infty}(H)\right]$ such that

(i) the map $\left(a, u, u^{\prime}\right) \rightarrow \phi_{n}(a)\left(u, u^{\prime}\right)$ is continuous from $G \times\left[D_{\infty}(H)\right]$ $\times\left[D_{\infty}(H)\right]$;

(ii) $\phi_{0}(a)\left(u, u^{\prime}\right)=\left\langle u, u^{\prime}\right\rangle$ for all $a \in G, \phi_{n}(a)\left(u, u^{\prime}\right)=0$ for all $a \in G$ and $n>0$;

(iii) denoting the sesquilinear form: $\left(u, u^{\prime}\right) \rightarrow \phi_{n}(a)\left(W(z) u, W(z) u^{\prime}\right)$ as $F_{n}(a, z)$, the following relations hold for real $x$ in $D_{\infty}(B)$;

$$
F_{j}(a, x)=F_{j}(a, 0), \quad F_{j}(a, i x)=\sum_{j=0}^{n}\left(\begin{array}{l}
j \\
k
\end{array}\right) F_{n-k}(a, 0) x(a)^{k} .
$$

The forms $\phi_{n}(a)$ may alternatively be interpreted as generalized operators, from the space $\left[D_{\infty}(H)\right]$ to its antidual; these operators in turn determine conventional operators on $K$, by restriction to those vectors whose transforms are again in $K$, where $K$ is injected into the antidual in the canonical fashion. Generally speaking, these forms are quite well behaved modulo the circumstance that the corresponding conventional operators typically have only 0 in their domains. A number of results in this connection may be illustrated by

TheOREM 4 [5]. If $G=R^{n}, n>1, B(k)=\left(m^{2}+k^{2}\right)^{1 / 2}, f \in L_{1}\left(R^{n}\right)$, and $\int \phi_{2}(a) f(a) d a$ maps (at least) one nonzero vector into $K$, then $f=0$.

THEOREM 5 [5]. If $f$ is a real element of $L_{1}(G)$ and $\int \phi_{n}(a) f(a) d a$ maps a dense domain in $K$ into $K$, then the corresponding conventional operator in $\boldsymbol{K}$ is essentially selfadjoint.

The strong regularizing effect of temporal smoothing is shown by the next result, which uses the notation $\phi_{n}(a, t)$ for $\Gamma(t) \phi_{n}(a) \Gamma(t)^{-1}$. 
THEOREM 6 [6]. If $B(\cdot) \in L_{p}\left(G^{*}\right)$ for all $p>3$, if $g \in L_{1}(G)$ and $g \in L_{1}\left(G^{*}\right)$, and $f$ is a real, even, element of $L_{1}\left(R^{1}\right)$ such that $f(\lambda)$ $=O\left(|\lambda|^{-n}\right),|\lambda| \rightarrow \infty$, then $\int \phi_{n}(a, t) g(a) f(t) d a d t$ maps all of $D_{\infty}(H)$ into $K$, and has a selfadjoint extension as an operator in $K$.

This result is essentially to the effect that as a conventional operator, $\int \phi_{n}(a, t) g(a) d a$ is a distribution in $t$ of order $n$. On restricting the form of the spectral function, e.g., to the relativistic case, slightly sharper results are obtainable as to the order, a point which is significant in connection with the integration of the associated partial differential equations (cf. [6]). In the relativistic case, for example, when $n=2, f$ may be a step function; for $n=3, f$ may have the form: $f(s)=1-|s| \epsilon^{-1}$ for $|s|<\epsilon, f(s)=0$ for $|s| \geqq \epsilon$.

3. The following brief physico-mathematical lexicon may be of use to some readers, and ignored by those of purely mathematical interests. Normal symmetric quantum process is an objective correlative for the variable term free Bose-Einstein quantum field, which is however usually used in connection with a specific partial differential equation. $K$ is the field state (vector) space; $\Psi(z)$ is a field operator (or variable); $\Gamma(U)$ is the second-quantized operator corresponding to the singly-quantized operator $U$, occurring in most treatments only in infinitesimal form and with $U$ restricted to be of the form $U(a)$ for some representation $U(\cdot)$ of the Poincare group; $v$ is the vacuum (state) vector. $H$ is the space of normalizable solutions of the abstract wave equation $\square \phi=0$, where $\square=(\partial / \partial t)^{2}-B^{2} ; A$ is the (singlyquantized) energy operator; $H$ is the (total) field energy operator.

\section{REFERENCES}

1. I. Segal, Notes toward the construction of non-linear relativistic quantum fields. I: The Hamiltonian in two space-time dimensions as the generator of a $C^{*}$-automorphism group, Proc. Nat. Acad. Sci. U.S.A. 57 (1967), 1178-1183. MR 35 \#5195.

2. - Nonlinear functions of weak processes. I, J. Functional Analysis 4 (1969), $404-456$.

3. - Foundations of the theory of dynamical systems of infinitely many degrees of freedom. I, Mat.-Fys. Medd. Danske Vid. Selsk. 31 (1959), no. 12, 39 pp. MR 22 \#3477. II, Canad J. Math. 13 (1961), 1-18. MR 23 \#B1877. III, Illinois J. Math. 6 (1962), 500-523. MR $26 \# 1075$.

4. - Local nonlinear functions of quantum fields, Proc. Conf. in Honor of M. H. Stone (Chicago, 1968) (to appear).

5. - Nonlinear functions of weak processes. II, J. Functional Analysis (to appear).

6. - Local non-commutative analysis, Proc. Sympos. in Honor of S. Bochner (Princeton, 1968) (to appear). 
7. G. C. Wick, The evaluation of the collision matrix, Phys. Rev. (2) 80 (1950), 268-272. MR 12, 380.

8. I. Segal, Interprêtation et solution d'équations non linéaires quantifiées, C. R. Acad. Sci. Paris 259 (1964), 301-303. MR 29 \#4453.

9. L. Gårding and A. S. Wightman, Fields as operator-valued distributions in relativistic quantum field theory, Ark. Fys. 28 (1964), 129.

10. I. Segal, Hypermaximality of certain operators on Lie groups, Proc. Amer. Math. Soc. 3 (1952), 13-15. MR 14, 448.

Massachusetts Institute of Technology, Cambridge, Massachusetts 02139 